\title{
VI. Ueber die absolute Anzahl von Schwingungen, welche zur Erzeugung eines Tones erforderlich sind; von Felix Auerbach.
}

Die Frage nach der absoluten Anzahl von Schwingungen, welche zur Erzeugung eines Tones erforderlich sind, zerfällt naturgemäss in zwei Fragen. Eine untere Grenze für die gesuchte $Z$ ahl ergibt sich nämlich ohne weiteres aus der Definition des Tonbegriffs, als der periodischen Wiederkehr irgend welcher Bewegungsform. Aus dieser Definition folgt nämlich, dass mindestens zwei Schwingungen erforderlich sind, und es bleibt nur noch die zweite Frage zu beantworten, ob diese Zahl auch in der That hinreichend sei.

Mit dieser Frage hat sich vor kurzem Hr. Pfaundler eingehend beschäftigt ${ }^{1}$ ) und ist schliesslich, auf Grund von Beobachtungen an der Sirene und mit Benutzung des sogenannten "tönenden Echos" zu dem Ergebnisse gelangt, dass in der That zwei Schwingungen zu dem gedachten Zweckeausreichen können. Den Bedenken, welche der $\mathrm{Hr}$. Verfasser selbst gegen die Strenge seiner Beweisführung äussert, glaube ich mich jedoch völlig anschliessen zu sollen, da bej den Erscheinungen, aus welchen Hr. Pfaudler den entscheidenden Schluss zieht, die Obertöne eine zu grosse Rolle spielen, als dass man sie unberücksichtigt lassen dürfte. Ich erlauhe mir daher, auf diese Frage hier zurückzukommen, und bemerke von vorn herein, dass meine Betrachtungen in der That zu einem erheblich grössern Werthe für die gesuchte Zahl führen.

Der vorliegenden Frage lässt sich von vier verscliedenen Seiten näher treten, von der direct physiologischen, von der psychologischen, von der physikalischen und endlich von der musikalischen. Was die erste betrifft, so kann ich mich hier kurz fassen, da eine Experimental-

1) Wien. Ber. 1878, II. Abth. 
untersuchung von Hrn. S. Exner') vorliegt, in welcher die Zabl siebzehn als die gesuchte aufgestellt wird, freilich in einer Bedeutung, welche mit der von Hrn. Pfaundler gemeinten sich vielleicht nicht völlig deckt. Zur Klarstellung dieses Punktes, d. h. zur genauen Feststellung des zu suchenden gehe ich zur psychologischen Behandlung der Frage über. Hr. Pfaundler hebt selbst die Nothwendigkeit hervor, zunächst den Sinn der gestellten Frage zu fixiren, geht aber, wie ich meine, in dieser Hinsicht nicht weit genug; er gibt zwar an, dass es bei der vorliegenden Untersuchung nicht um den physikalischen Begriff des Tones, sondern nur um den physiologischen sich handeln könne, er unterscheidet zwar weiterhin zwischen einem Percipiren und einem Appercipiren des Tones; aber auf den für die Sache wesentlichsten Punkt geht er nicht ein. Ton im physiologischen Sinne und Ton im physikalischen Sinne stehen in der Beziehung zu einander, dass ersterer die Empfindung des letztern ist. Befindet sich in einem Raume, in welchem letzterer entsteht, ein Ohr, so entsteht auch ersterer; aber nicht gleichzeitig mit jenem, sondern durch eine messbare, wenn auch kleine Zeit von ihm getrennt. Geht man nun daran, diese Zeit zu bestimmen, so gelangt man zu der Erkenntniss, dass hier zwei verschiedene Zeiten gemeint sein können, welche getrennt behandelt werden müssen, nämlich die Zeit, welche von dem Momente des Entstehens des physikalischen Tones vergeht, bis die Empfindung eines Tones entsteht, und die Zeit, welche von demselben Momente verstreicht, bis der Ton als solchor erkannt wird. Dass zwischen beiden Zeiten ein Unterschied besteht, und wie man sich diesen Unterschied vorzustellen habe, ergibt sich aus einer einfachen Versuchsmethode. Die erstere Zeit erhält man nämlich, wenn man jemand auf einen bestimmten, unveränderten und oft nacheinander ausgeübten Gehörsreiz von der Natur eines einfachen Tones

1) Pflüger's Archiv XIII. p. 228. 1876. 
reagiren lässt und den Mittelwerth aller erhaltenen Empfindungszeiten nimmt. Die letztere Zeit dagegen erhält man, wenn man dem Betreffenden aufgibt, von verschiedenen ihm vorher bekannt gemachten, an Stärke oder Höhe oder Klang ungleichen Tönen, welche in unregelmässiger Folge ihm zu Gehör gebracht werden, nur auf einen einzigen, vorher ausgewählten zu reagiren, und wenn man dann den Mittelwerth aus allen erhaltenen Unterscheidungszeiten zieht. In der That ergibt sich die Unterscheidungszeit grösser als die Empfindungszeit.

Bei Gelegenheit einer von Hrn. J. v. Kries und mir ${ }^{1)}$ ausgeführten Bestimmung beider Zeiten für verschiedene Sinnesorgane bot sich die Möglichkeit, auf diese Weise die Anzahl der zur Erzeugung einer Tonempfindung erforderlichen Schwingungen zu bestimmen. In Bezug auf die Versuchsanordnung erlaube ich mir auf die Originalabhandlung zu verweisen und bemerke nur, dass die ziemlich reinen Töne, auf welche sich die folgenden Zahlenangaben beziehen, von Stahlplättchen erzeugt wurden in dem Momente, wo diese von zwei unter ihnen angebrachten Electromagnetpolen infolge Verschwindens des Magnetismus abrissen. Die Schwingungszahlen der Töne waren resp. $640,500,400$ in runden Zahlen; dem electrischen Funken entspricht nach Hrn. Toepler's Versuchen ein einziger Impuls. Es zeigte sich nun folgende doppelte Erscheinung, welche bei anderen Sinnesorganen nicht entsprechend auftrat. Erstens ergab sich die einfache Empfindungszeit als abhängig von der Tonhöhe und grösser als die entsprechende Zeit bei dem Geräusche des electrischen Funkens, wie folgende Tabelle zeigt:

Empindungazeit in Secanden

(im Mittel aus den fur beide Experimentirende erhsitenen Werthen).

\begin{tabular}{c|c|c|c}
\hline \hline Tiefater & Mittlerer & Höchster & Funke \\
\hline 0,158 & 0,154 & 0,141 & 0,130
\end{tabular}

1) Arch. f. Auat. u. Physiol., von $\mathrm{His}$, Braune und Du Bois. Reymond, p. 297-378, ef. p. 326 ff. 1877.

Ann. d. Phys. u. Chem. N. F. VI. 
Aus der Verschiedenheit dieser Zahlen ist, wie wir glaubten, der Schluss zu ziehen, dass einige Schwingungen vergehen müssen, ehe überhaupt eine Tonempfindung entsteht. Hierfür ergibt sich, wenn die Differenzen obiger Zahlen mit den betreffenden Schwingungszahlen multiplicirt werden, im Mittel die Zahl 10. Die einzelnen Zahlen $(7,12,11)$ schwanken zwar ziemlich erheblich; indess sind die Abweichungen, in Anbetracht der schwierigen Versuchsverhältnisse und ihrer regellosen Anordnung, nicht so gross, dass man nicht schliessen dürfte: die gefundene $Z$ ahl sei von der Tonhöhe wenig oder gar nicht abhängig.

Zweitens zeigte sich bei den Versuchen über Unterscheidung zwischen einem hohen und einem tiefen Ton, dass das Erkennen des hohen Tones schneller erfolgt als das Erkennen des tiefen. Diese Erscheinung ist von der ersten völlig verschieden. Die eigentliche Unterscheidungszeit, als Differenz zweier Empfindungszeiten, kann daron durchaus nicht abhängig sein, dass die Tonempfindung Zeit zum Entstehen braucht. Es ist vielmehr zu schliessen, dass, nachdem die Tonempfindung zu Stande gekommen ist, wiederum noch einige Schwingungen vergehen müssen, ehe diese Tonempfindung ihre charakteristische Tonhöhe erbält. Um die Anzahl $x$ dieser Schwingungen zu ermitteln, nenne ich $y$ die Unterscheidungszeit, welche sich unabhängig von der Tonhöhe ergeben wurde, wenn die Tonempfindung von Anfang an ihre bestimmte Tonhöhe besässe, $n_{t}$ und $n_{T}$ die beiden Schwingungszahlen, $U_{t}$ und $U_{T}$ die entsprechenden Unterscheidungszeiten; dann gelten die Gleichungen:

$$
\frac{x}{n_{t}}+y=U_{t}, \quad \frac{x}{n_{T}}+y=U_{T},
$$

also:

$$
x=n_{t} n_{T} \frac{U_{t}-U_{T}}{n_{T}-n_{t}} .
$$

Es war $n_{t}=400, n_{T}=640$, und es fand sich im Mittel von vielen Einzelwerthen:

$$
U_{t}=0,044 \mathrm{sec}, \quad U_{T}=0,034 \mathrm{sec} .
$$


Hieraus ergibt sich $x$ als zwischen 10 und 11 gelegen. Addirt man hierzu die vorhin gefundene $\mathrm{Zahl}$, so gelangt man zu dem Ergebniss, dass etwa 20 Schwingungen erforderlich sind, bis die Empfindung nicht nur entstanden ist, sondern auch ihren ausgeprägten Charakter erhalten hat. Dieses Resultat stimmt mit dem Ergebniss der Untersuchung des Hrn. Exner sehrt gut überein.

Vom physikalischen Standpunkte drittens ist es, wie schon erwähnt, wohl ohne weiteres erklärlich, dass eine einzige Schwingung nicht genligt, um eine Tonempfindung zu erzeugen; aus welchem Grunde aber sollen deren mehr als zwei nothwendig sein? Ich glaube, den Grund in dem Umstande zu erblicken, dass der Vorgang des Hörens, wie jeder Vorgang des Mitschwingens, nicht den Charakter eines plötzlichen Herausgreifens, sondern den einer allmählichen Auslese hat. Denken wir uns den Aufhängepunkt eines Pendels in Schwingungen versetzt. Die erste Schwingung versetzt die Pendelkugel, gleichviel welche Länge der Faden besitzt, in Schwingung in derselben Ebene; die zweite fördert diese Schwingungen, falls sie um die Schwingungsdauer der Pendelkugel später als die erste erfolgt; sie vernichtet sie, falls sie um die Hälfte der Schwingungsdauer der Pendelkugel später als die erste erfolgt; in allen anderen Fällen tritt eine allmähliche Schwächung der Schwingungen der Pendelkugel ein. Aehnlich verbält es sich beim Vorgange des Hörens. Die erste dem Obre zugeführte Schwingung erregt alle Corti'schen Fasern; die zweite bringt nun nicht sofort alle zur Ruhe, bis auf diejenigen, welche dauernd in der Periode des betreffenden Tones schwingen können, weil der Unterschied beider Perioden null oder sehr klein ist, sondern sie gestattet auch noch vielen, zu beiden Seiten der obigen gelegenen Fasern, weiter zu schwingen, etwa allen denen, welche seit dem ersten Impulse mehr als das $\left(1-\frac{1}{2 k}\right)$ fache und weniger als das $\left(1+\frac{1}{2 k}\right)$ fache einer Schwingung been- 
det haben. Einen physikalischen Ton dieser Art will ich einen Ton von der Breite $k$ nennen. Aus der Art und Weise, wie die Bewegung der ausschwingenden Fasern durch jeden neuen Impuls beeinflusst wird, ergibt sich, dass die Breite des Tones nach der dritten Schwingung nur noch $\frac{k}{2}$, nach der vierten $\frac{k}{3}$ u. s. w., nach der $p$ ten nur noch $\frac{k}{p-1}$ beträgt. Um zu ermitteln, wieviel Schwingungen $p$ erforderlich seien, um einen Ton zu erzeugen (d. h. einen physiologischen Ton, eine Tonempfindung), hat man also 1) die Breite des durch den zweiten Impuls erzeugten physikalischen Tones $k$ und 2) die Breite $k^{\prime}$ zu ermitteln, welche ein physikalischer Ton besitzen darf, um einen physiologischen Ton zu erzeugen, welchem ein reiner ausgeprägter Charakter zukommt. Bei der Unbestimmtheit der Aufgabe und der unbekannten Natur der Corti'schen Gebilde lässt sich in Bezug auf die erste Zahl nur eine ganz rohe Schätzung machen. Berücksichtigt man Schwingungen bis $\mathrm{zu} \frac{1}{10}$ der maximalen Intensität und benutzt man die Analogie, welche Pendelschwingungen mit Tonschwingungen besitzen, so findet man, dass man etwa $k=15$ bis 25 Procent, also rund $k=0,20$ setzen kann. Andererseits müsste $k^{\prime}$ erst noch durch bezügliche Versuche ermittelt werden; aus leicht sich ergebenden musikalischen Gründen lässt sich aber mit grosser Wahrscheinlichkeit schliessen, dass $k^{\prime}$ kleiner sei als die dem sogenannten Komma (80:81) entsprechende relative Differenz der Schwingungszahlen ( $\left(\frac{1}{80}\right)$, und zweitens, dass es nicht beträchtlich kleiner sei. Setzt man demgemäss rund $k^{\prime}=0,01$, so erhält man als die gesuchte Zahl $p=\frac{k}{k^{\prime}}=20$.

Für den auf drei völlig verschiedenen Wegen erhaltenen Werth von etwa 20 Schwingungen, welche vergehen müssen, ehe eine charakterisirte Tonempfindung entsteht, kann man noch auf einem vierten Wege, von der musikalischen Seite, eine Bestätigung suchen. Auf diese Möglichkeit hat Hr.Pfa und er zwar in der Einleitung seines Aufsatzes aufmerksam gemacht, ist aber nicht wieder darauf zu- 
rückgekommen. Und nicht mit Unrecht. Spielen doch gerade bei den in der Musik gebräuchlichen Tönen die Obertöne die wesentlichste Rolle. Aber es ist leicht einzusehen, dass der Einfluss der Obertöne sich nur derart geltend machen kann, dass die zu bestimmende Zahl zu klein ausfällt; dieselbe wird also immerhin die Bedeutung einer untern Grenze haben. Die musikalische Thatsache, um welche es sich handelt, ist die, dass Triller und Vorschläge in tiefen Lagen verschmäht oder, wo sie noch vorkommen, schwerfälliger werden. Diese Thatsache erklärt sich in den meisten Fällen wohl am einfachsten aus der Rücksicht auf das störende Nachklingen der Töne. Versucht man es, sie durch den Umstand zu erklären, dass eine gewisse Anzahl von Schwingungen zur Erzeugung und Charakterisirung des Tones erforderlich sind, so findet man hierfür etwa die Zahl 10 als untere Grenze.

Aus alledem ergibt sich, dass wahrscheinlich etwa zwanzig Schwingungen zur Erzeugung eines charakterisirten Tones orforderlich sind.

Breslau, 23. Jan. 1879. ')

\section{Ueber die Emissionsspectra der Haloäd- verbindungen des Quecksilbers; von B. O. Peirce.}

Die Emissionsspectra der Quecksilberhalö̈dverbindungen erhält man am bequemsten, wenn man den Strom eines Inductoriums durch eine Geissler'sche Röhre leitet, in die eine kleine Menge des Salzes gebracht ist, und die man dann erhitzt. Ist die Röhre kalt, so tritt das bekannte Luftspectrum auf. Erwärmt man sie langsam mit einem Bunsen'schen Brenner, so verschwindet dasselbe und das gewöhnliche Quecksilberspectrum erscheint. Bei weiterem Erwärmen tritt eine für jedes Salz verschiedene Lichtbande auf.

1) Wie ich nachträglich erfahre, hat auf andere Weise auch Hr. Mach (Lotos, Aug. 1873), sogar ohne Gewähr dea Ausschlusses von Obertönen, die Zahl 4 bis 8 als Minimum gefonden. 